Interacciones que transforman:

\title{
Implementación de Comunidades de Aprendizaje en el Perú durante los años 2014 - 2018
}

Interactions that transform: Implementation of Learning

Communities in Peru during the years 2014 - 2018

Mg. Roberto Barrientos Mollo

Universidad Marcelino Champagnat

rbarrientosm@umch.edu.pe
Recibido el 04/01/2019

Revisión del 14/01 al 30/05/2019

Publicación 16/07/2019

\section{Resumen}

El presente artículo es un esfuerzo de sistematización y análisis de las teorías de la acción utilizadas en el proyecto Comunidades de Aprendizaje entre los años 2015-2018 en el Perú. También se realiza un análisis del proceso de escalonamiento en torno a cuatro categorías: profundidad, sostenibilidad, difusión y empoderamiento. Los resultados muestran que existe un mayor empoderamiento y difusión cuando se utiliza un enfoque de movimiento social, así como un enfoque de trabajo en redes fortaleciendo el trabajo de los directores y entre directores, según las zonas geográficas en las que se encuentran adscritos. El artículo concluye que se han realizado avances en estas cuatro categorías; sin embargo, el cambio educativo es altamente incierto y es un proceso lento; el ejercicio de explicitación y revisión continua de la teoría de la acción ha sido útil para la mejora de la implementación. Se espera que sea un aporte para implementadores de programas educativos, como directores y docentes.

Palabras clave: Comunidades de aprendizaje, escalonamiento, política pública, mejora escolar. 


\section{Summary}

This article is an effort to systematize and analyze the theories of action used in the Communities of Learning project between 2015-2018 in Peru. Likewise, an analysis of the staging process is made around four categories: depth, sustainability, diffusion and empowerment. The results show that there is greater empowerment and diffusion when a social movement approach is used, as well as a focus on networking, strengthening the work of the directors and among directors, according to the geographical areas in which they are attached. The article concludes that progress has been made in these four categories; however, educational change is highly uncertain and is a slow process; also, the exercise of explicit and continuous review of the theory of action has been useful for the improvement of the implementation. It is expected to be a contribution for implementers of educational programs, such as directors and teachers.

Key words: Communities of learning, scaling, public policy, school improvement. 


\section{Introducción}

En el debate de las políticas públicas está siempre presente la idea de escalonamiento de propuestas innovadoras. Sin embargo, la investigación muestra que dicho escalonamiento y sostenibilidad presentan serias dificultades (Bryk, Sebring, Allensworth, Luppescu, y Easton, 2010; Farrell, Manion, y Rincón-Gallardo, 2017). En la actualidad, en el Perú se vienen implementando diversas reformas y propuestas de cambio que buscan transformar las prácticas docentes, lo que hace necesario generar un espacio de reflexión sobre lo que se entiende por escalonamiento y sus condiciones de calidad, pues, tanto en lo privado como en lo público, surge la inquietud sobre cómo lograr más cobertura sin perder la calidad. Para el presente trabajo se utilizan cuatro categorías para analizar el crecimiento o expansión del proyecto Comunidades de Aprendizaje: difusión, expansión, empoderamiento y sostenibilidad (Coburn, 2003) (ver figura 1). Muchas intervenciones, que buscan un cambio en el aula que genere resultados de aprendizaje, desaparecen cuando la presión o recursos de la intervención desaparecen.

ENFOQUE UNIDIMENSIONAL

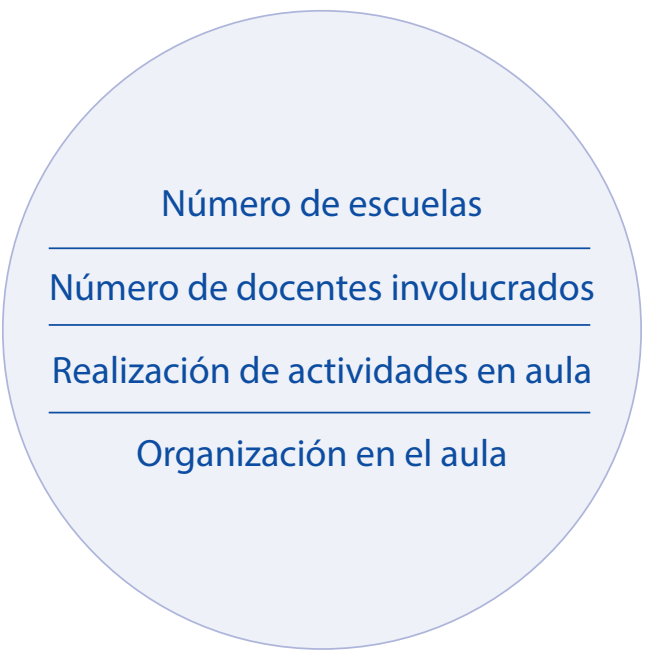

\section{ENFOQUE MULTIDIMENSIONAL}

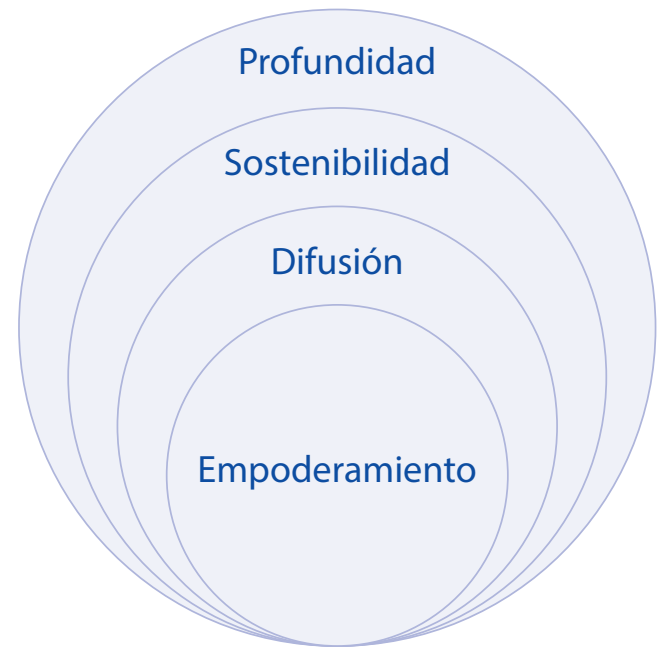

Figura 1. Enfoques de expansión con calidad. Elaboración propia a partir de Coburn 2003. 
Es necesario empezar con una definición de escala. Stringfield y Datnow definen el escalonamiento como "la expansión deliberada a muchos escenarios, de un diseño de reestructuración de la escuela desarrollado externamente, que previamente se ha utilizado con éxito en uno o un pequeño número de escuelas “ (1998, p. 271). Se entiende por "diseño de reestructuración", cualquier tipo de propuesta de intervención o innovación que se desea introducir en una o más escuelas. En opinión de Coburn, esta definición es incompleta, puesto que no toma en cuenta el cambio en profundidad (2003). Por ello, propone cuatro dimensiones para entender y ejecutar el escalonamiento: profundidad, sostenibilidad, difusión y empoderamiento de la propuesta de parte de los actores.

\section{Dimensiones del escalonamiento}

En la tabla 1, se presenta una síntesis de cada dimensión, es decir, su definición y preguntas que pueden servir para la reflexión y la discusión.

Dimensión

Profundidad
Definición

Es lograr una transformación

profunda en las escuelas, mediante el cambio en las creencias docentes, las normas de interacción social y los principios pedagógicos que se quiere difundir. En esta dimensión, la variación no se reduce a la entrega de materiales o adición de actividades en el aula.
Preguntas para la reflexión

¿El encuentro de los maestros con la propuesta que se quiere introducir les hace repensar y reconstruir sus creencias o, simplemente, ellos alteran la propuesta de manera que refuerzan sus supuestos y prácticas preexistentes? 
Dimensión

Sostenibilidad
Definición

Se refiere a la duración de la propuesta en el tiempo y al conjunto de estrategias para lograrlo.
Preguntas para la reflexión

¿Qué estrategias son efectivas para desarrollar y fomentar la profundidad en la ejecución de la propuesta por parte de los docentes? ¿Cómo pueden los implementadores trabajar para crear las condiciones claves en las escuelas y gobiernos de tal manera que apoyen y mantengan el cambio en el aula a lo largo del tiempo?
Es el conjunto de acciones para difundir las creencias, normas y principios subyacentes de la propuesta a otras aulas y escuelas.
¿La autoridad local protege y respeta las actividades de las escuelas que aplican la propuesta y/o las toma como referentes, para toda su jurisdicción?

¿El distrito o provincia realiza acciones desde la normativa, espacios de desarrollo profesional y rutinas escolares que faciliten la propagación y profundización en la propuesta? 
Dimensión

Empoderamiento
Definición

Se refiere al proceso de apropiación de la propuesta por parte de los líderes distritales, escolares y docentes.
Preguntas para la reflexión
¿Qué estrategias son eficaces para desarrollar las capacidades necesarias para la apropiación de la propuesta? ¿Son diferentes estrategias para cada nivel del sistema (aula, escuela, distrito, nación)? ¿Cómo pueden los implementadores sentar las bases para un cambio de lo externo a lo interno desde los primeros días de compromiso con una escuela o distrito?

tabla 1. Dimensiones de la escala. Elaboración propia a partir de Coburn, 2003.

Se puede ensayar una definición multidimensional de escala como la expansión deliberada a muchos escenarios educativos de una determinada propuesta que transforma creencias, maneras de interactuar entre docentes y estudiantes; se hace sostenible en el tiempo mediante una apropiación efectiva de parte de los actores. 
últimos son capaces de "demostrar una

Desde el ámbito de la gestión del cambio se ha desarrollado un marco teórico sobre las teorías de la acción. Por teoría de la acción se entiende al conjunto de ideas y/o supuestos que guían una determinada acción o estrategia de cambio. Para algunos es un "guión que aterriza una visión y estrategia" (City, Elizabeth, Elmore, Fiarman, y Teitel, 2009). Detrás de toda intervención, existe una determinada teoría de la acción. Para Ries, esta se entiende como una hipótesis sobre las cosas que deben o debieran ocurrir si se aplican determinadas acciones (2011). Los requisitos para una buena teoría de la acción son los siguientes: 1) partir de un predicamento de relación causal (Si... entonces), 2) tiene que ser empíricamente refutable $y, 3$ ) tiene que estar abierta a revisiones y mejoras (City, Elizabeth et al., 2009). El primer requisito se refiere a la verbalización o redacción que permita conocer y visualizar la cadena de supuestos de cambio. El segundo requisito, plantea el desarrollo de un conjunto de indicadores que permitan evaluar de manera continua la falsedad o no de la hipótesis; Ries recomienda trabajar con indicadores accionables (2011). Estos relación de causa-efecto" (Ries, 2011, p. 89). Esta revisión periódica de las hipótesis de cambio permite ofrecer sustento para realizar las revisiones y mejoras necesarias (requisito 3 ). Sin embargo, el requisito tres presenta un nivel de dificultad mayor, puesto que las personas tienden a defender y aferrarse a sus ideas, y pueden ser capaces de racionalizar una intervención a pesar de existir suficientes evidencias de su fracaso. Ello lleva al momento clave en todo proceso de implementación, la decisión entre pivotear o perseverar. Para Ries (2011), pivotear es "una corrección estructurada para probar una nueva hipótesis sobre el producto, la estrategia o el motor de crecimiento" (p. 140).

De lo que se trata es de aprender haciendo y "realmente aprender pensando continuamente en el hacer" (Fullan y Hargreaves, 2009, p. 96). Es por ello que "se debe de construir una constante evaluación e indagación dentro de la mentalidad de la reforma, ya sea en el equipo central que lidera la reforma como en las personas que están en el campo. Se espera que los líderes de todos los niveles tengan una disposición inquisitiva: ¿Estamos 
implementando la estrategia de forma efectiva? ¿Está funcionando? ¿Hay alguna sorpresa? ¿Qué estamos aprendiendo?" (Fullan, 2009, p. 67). Se puede afirmar que la construcción de la teoría de la acción; implica mucho aprender de la misma acción. Es por ello que el testeo o experimentación es parte muy importante de todo proceso de innovación, puesto que, a "diferencia de la planificación estratégica tradicional, esta especificación estará enraizada en la retroalimentación de lo que está funcionando hoy, en lugar de lo que se anticipa que puede funcionar mañana" (Ries, 2011, p. 142). En la medida en que se tenga una teoría de la acción explícita, refutable y abierta a revisiones tendrá más posibilidades de tener resultados positivos.

\section{Contexto}

En el año 2013, el Instituto Natura, fundación de la empresa Natura Cosméticos, decide implementar el proyecto de transformación educativa y social "Comunidades de Aprendizaje" en Latinoamérica, específicamente en Brasil, Argentina, Chile, México, Colombia y Perú. El Instituto Natura tiene como visión crear las condiciones para que todos los ciudadanos formen una comunidad de aprendizaje y contribuir de esa manera con el cierre de brechas en educación; en ese sentido busca que se garantice un desarrollo humano sostenible en el planeta. El proyecto Comunidades de Aprendizaje ha sido desarrollado por investigadores del Community of Research on Excellence for All (CREA) de la universidad de Barcelona en España.

A continuación, se desarrollan las teorías de la acción que se han manejado en los últimos años para la implementación de Comunidades de Aprendizaje y los respectivos cambios realizados fruto de la reflexión sobre la acción. Antes, es necesario explicitar la teoría de la acción general del proyecto:

Si se viven los siete principios del aprendizaje dialógico, por medio de la ejecución amplia y frecuente de las siete Actuaciones Educativas de Éxito y las cinco fases de transformación, entonces los aprendizajes y la convivencia escolar mejorarán. 
A partir de esta teoría de la acción, cada país desarrolla su propia teoría de la acción para los procesos de implementación del mismo, adaptándolo a las diversas realidades y contextos donde toca implementar.

\section{Breve explicación del proyecto}

El proyecto consiste en la inserción progresiva en la vida escolar del enfoque del aprendizaje dialógico, un concepto de aprendizaje que consiste en la involucración de todos aquellos que son parte de la vida del estudiante, para mejorar sus aprendizajes: escuela, familia y comunidad (Aubert, Flecha, García, Flecha y Racionero, 2011). Dicho concepto se desagrega en siete principios (solidaridad, transformación, inteligencia cultural, igualdad de diferencias, diálogo igualitario, dimensión instrumental y creación de sentido) que encuentran su concreción en siete estrategias y cinco momentos de movilización escolar llamados "fases de transformación" (Valls, 2000). El enfoque filosófico del proyecto está basado en la pedagogía popular de Freire (2005) y en la teoría de la Acción Comunicativa del filósofo Jurgen Habermas (1987), que propone que la palabra tiene una fuerza transformadora. Para diversos autores se está viviendo en la sociedad un "giro dialógico", es decir, un cambio en las relaciones entre personas y entre instituciones, que tiene entre sus características un trato más igualitario (Alonso y Saso, 2011; Aubert, García, y Racionero, 2009; Habermas, 1987). Las ideas y estrategias centrales del proyecto fueron gestadas por el investigador Ramón Flecha (Aubert et al., 2011). A fines de los años 60, Ramón Flecha se fue a vivir a las chabolas en una zona deprimida de Barcelona, en las que inició un trabajo social intenso. Este trabajo estuvo basado en la inclusión de los inmigrantes y personas desfavorecidas de España. Una de sus principales iniciativas fue crear un centro de formación de adultos llamado la Verneda - Sant Martí, llamado "Escuela de Personas Adultas de la Verneda". Esta es considerada como la primera "comunidad de aprendizaje". Posteriormente, se iniciarían las experiencias en escuelas y se irían desarrollando las estrategias del proyecto. En el año 2010, se realizó una investigación para ver las mejores prácticas que generen aprendizajes e inclusión social, $y$, de esta manera, se terminaron de consolidar las siete estrategias del proyecto, llamadas Actuaciones Educativas de Éxito (Flecha, 2006, 2010). El proyecto se fue consolidando hasta ser conocido por el Instituto Natura que decide implementarlo en Latinoamérica. 


\section{Desarrollo de la experiencia}

Los primeros años: 2013-2016.

En el marco de esa teoría de la acción, se desarrollaron en el Perú estrategias específicas. A continuación, se explica el proceso de implementación. La teoría de acción específica se realizó bajo las siguientes acciones: se empoderó a aliados educativos específicos, se inició un acompañamiento básico y una estrategia de capacitación, se inició un proceso de crecimiento con calidad (se puede ver la explicación gráfica en la figura 1) se realizaron acciones iniciales, entre ellas, presentar el proyecto a posibles interesados. En octubre del año 2013, se realizó el primer Foro Internacional en el Perú, que tenía como objetivo presentar el proyecto e invitar a primeros operadores. En ese momento, manifestó su interés la Dirección Regional de Lima Metropolitana, oficina de gobierno subnacional de Lima Metropolitana y la Organización No Gubernamental Prorural, entidad que vela por el cierre de brechas en las zonas rurales mediante su modelo de Formación en Alternancia. El acuerdo fue iniciar un piloto de implementación con pocas escuelas para una posterior escalabilidad. Se eligieron 7 escuelas para iniciar en el 2014, de las cuales tres estaban en Lima y cuatro en provincias, 2 en Cusco y 2 en Piura. En la tabla 2 se muestra el proceso de crecimiento de las escuelas involucradas en el proyecto el Perú a lo largo de los años.

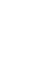


Barrientos,R (2019) Implementación de comunidades

de aprendizaje en el Perú años 2014-2018

Revista Educa UMCH,13(1). PP.38-62.

https://doi.org/10.35756/educaumch.v0i13.94

\begin{tabular}{cc}
\hline Año & Número de escuelas \\
\hline 2014 & 7 \\
\hline 2015 & 27 \\
\hline 2016 & 42 \\
\hline 2017 & 52 \\
\hline 2018 & 60 \\
\hline
\end{tabular}

Tabla 2. Número de escuelas involucradas en el proyecto.

La teoría de la acción para el proceso de implementación fue la siguiente:

SI se acompaña mensualmente con un empoderamiento con acento individual y se realiza una estrategia informativa con la UGEL e involucra al gobierno nacional, se garantizará una implementación con calidad y bases para la sostenibilidad.

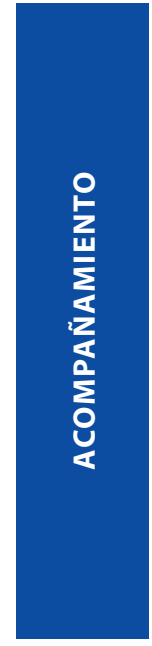

Visitas mensuales

Empoderamiento básico al director

Fortalecimiento de la UGEL con una visita informativa de inicio de año

\section{Generación}

de normativas

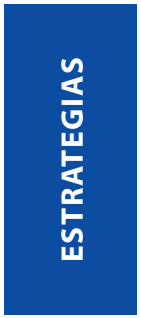

\section{Exposiciones}

Pasantías como estrategia para formadores locales

Figura 2. Explicación gráfica de la teoría de la acción 2014-2016

La estrategia inicial en el año 2014 para el desarrollo de capacidades, consistió en la selección de un grupo de docentes líderes con los respectivos directivos para participar de un programa de capacitación en la universidad de Barcelona, en el que estuvo incluida una pasantía a escuelas referentes en España. En este programa de capacitación también 
participaron docentes y directivos del Instituto Pedagógico Nacional de Monterrico, una entidad referente de formación docente en Perú. Como parte del programa de capacitación se recibió un entrenamiento in situ en Lima, Piura y Cuzco por tres investigadores de la Universidad de Barcelona para capacitar a las siete escuelas piloto, el que consistía en conferencias acerca de la propuesta. Así mismo, se hizo entrega de bibliotecas de libros clásicos de la literatura para la realización de una de las siete estrategias del proyecto y la tertulia literaria. En los años 2014 y 2015, se realizó un tipo de acompañamiento esporádico basado en la visita periódica a las escuelas seleccionadas con el fin de apoyar y acompañar el proceso; el equipo en ese momento estaba formado por dos personas. Los supuestos que estuvieron detrás del proceso de acompañamiento fueron los siguientes: si se realiza una visita esporádica, se observa la realización de la estrategia en el aula y se conversa con el docente, se activarán adecuadamente las estrategias del proyecto. Se manejó este supuesto hasta fines del 2015. En el proceso, se comprobó que para una adecuada implementación del mismo era necesario involucrar a otros actores, es por ello que se revisó y afinó la teoría de la acción. A fines del año 2015, se realizó una primera expansión a las escuelas de la red de las mismas, aliadas del Instituto Pedagógico Nacional de Monterrico (8) y las escuelas de la red de Prorural (12).

Aprendizajes del año 2014- 2015

\section{Condiciones favorables}

\section{para la implementación}

Se encontró como aspecto favorable que las escuelas con poblaciones medianas (de hasta 600 estudiantes) permitían una implementación más rápida, al mismo tiempo que se podía movilizar a toda la escuela. Mientras que escuelas con poblaciones elevadas y distintos turnos de atención (mañana y tarde) presentaban demoras en las fases de transformación debido a falta de participación, consensos y asistencia a reuniones.

Uno de los aspectos favorables para la implementación e impulso del proyecto fue el liderazgo del director. En ese momento, se entendía liderazgo como la capacidad del directivo de movilizar a la escuela hacia metas específicas. Las características encontradas fueron las siguientes: empoderamiento en el proyecto, directores designados por períodos de gestión de más 2 años, involucración efectiva en los temas 
pedagógicos, delegación de asuntos menos importantes. Desde el lado de los docentes, contribuyó a la buena implementación contar con equipos de docentes con bajo nivel de rotación.

\section{Condiciones desfavorables}

Las escuelas que contaban con directivos sin permanencia, sin interacción en el área pedagógica o compromiso con el proyecto tendían a no realizar o hacer más lento el proceso de implementación. Las escuelas con alta rotación de docentes en cada año, como sucedía en los centros de práctica del IPNM donde, en algunos casos, el $100 \%$ de docentes cambian año a año, o en los Centros Rurales de Formación en Alternancia donde se rota al $90 \%$ de docentes, la adopción era bastante difícil.

Las estrategias de capacitación para todos los docentes en ese momento eran del tipo expositivo con algunas dinámicas intermedias y se realizaban al inicio del año escolar. A este conjunto de acciones se les llama "sensibilización". Se vio que eran poco efectivas para la apropiación de las estrategias. Se evidenció que para una mejor implementación de las estrategias existían mayores posibilidades de permanencia, si es que se las insertaba en la dinámica habitual de la escuela; es decir, con espacios y tiempos definidos dentro del horario escolar y con indicaciones generales para todos los docentes. Se observó en ese momento que la implementación de estrategias fuera del horario escolar o aquellas realizadas solo por un grupo pequeño de docentes tendían a ser menos sostenibles.

Las relaciones con los representantes del órgano de gobierno más cercano a la escuela, la Unidad de Gestión Educativa Local, eran escasas, dado que la relación inicial se realizó con la Dirección Regional de Educación de Lima Metropolitana (DRELM). Estas relaciones consistían en una reunión de inicio de año, en la que se otorgaba una explicación sobre el trabajo a realizarse.

En el año 2016, basados en recomendaciones de Hargreaves y Fullan (2014; 2011), sobre el desarrollo de capital social, empezaron a realizarse pasantías entre las escuelas que aplicaban el proyecto $y$, como estrategia de presentación del mismo, a otras escuelas. Esta generaba mayor convencimiento, empoderamiento y claridad sobre lo que significaba trabajar en la misma.

El trabajo de incidencia política se realizaba con el Ministerio de Educación 
del Perú, con los responsables de oficinas relacionadas con las temáticas del proyecto, como son la Dirección General de Educación Básica Regular, encargada de diseñar e implementar el currículo, la Dirección General de Servicios Especializados y del ámbito rural (DIGEIBIRA), responsable del trabajo en los Centros Rurales de Formación en Alternancia (CRFA) y la Dirección General de la Calidad Educativa (DIGC), encargada de la gestión escolar. Dicho trabajo de incidencia consistía en presentar la propuesta y encontrar puntos de interés con los responsables de dichas oficinas. Los resultados fueron positivos en el caso de la Dirección de Servicios Educativos del Ámbito Rural, órgano de línea que depende de la DIGEIBIRA, por su interés en insertar, de manera permanente, las estrategias del proyecto en las escuelas a su cargo. Un factor que hacía difícil el trabajo coordinado y articulado con dichas oficinas es la alta rotación existente en el Ministerio de Educación. Por ejemplo, se dio el caso de una oficina que contó con cinco jefes distintos en un año.

\section{Teorías de la acción 2017- 2018}

Con los aprendizajes previos se decidió reformular la teoría de la acción con el siguiente predicamento:

\section{SI se desarrollan culturas}

colaborativas intra y extra escuela, desarrollo lateral de capacidades (Fullan, 2005), basado en el empoderamiento de los actores mediante una estrategia topdown y bottom-up, se garantizará una implementación con calidad y bases para la sostenibilidad. 


\section{Estrategias Bottom up}

\section{Visitas semanal / \\ quincenal / mensual}

Red de directivos

por cluster

Coaching a

los directivos

Fortalecimiento

de la UGEL

Generación de

normativas

Programa de certificación en Comunidades de Aprendizajes por 200 horas

Foro Nacional

Pasantías a escuelas de red

Resensibilizaciones

La Figura 3. Grafica los principales elementos de la teoría de la acción del período 2017-2018.

\section{La estrategia de copo de nieve}

Basados en las recomendaciones y experiencia de Rincón- Gallardo (2012), se eligió seguir el enfoque de cambio educativo como movimiento social (Colbert y Sarmiento, 2017), dado que garantiza mayores niveles de sostenibilidad al no apoyarse solo en las normativas, sino en la acción colegiada y voluntaria de docentes y funcionarios públicos. Para ello, se siguieron los enfoques y estrategias planteadas por el teórico de los movimientos sociales Ganz (2009, 2015). Entre las estrategias planteadas por Ganz está la estrategia de "copo de nieve", que consiste en trabajar en red a manera de un copo de nieve, que se concibe como "prácticas de liderazgo por medio del desarrollo de otros líderes, quienes, a cambio, desarrollan a otros líderes" (Ganz 2015, page 15). La definición de liderazgo de Ganz es "aceptar la responsabilidad de ayudar a otros a cumplir sus objetivos en condiciones de incertidumbre" (2015, p. 12). Si bien la estrategia es orientada a los movimientos sociales, contribuye con una mirada muy cercana a lo que ocurre en las escuelas públicas. Es importante entender que no solo consiste en distribuir el liderazgo entre un grupo de 
personas; cuando hablamos de líderes interconectados, hablamos de procesos que han ocurrido, previamente, como es el de tener un propósito común que los une y una corresponsabilidad que permite que asuman desafíos colaborativamente. La estrategia del copo de nieve utilizada en Comunidades de Aprendizaje no solo es un acelerador en el proceso de implementación y de apropiación del proyecto en la escuela, sino que contribuye con la sostenibilidad del proyecto, ya que, cada uno de los primeros líderes de un equipo está pensando en formar y desarrollar las habilidades necesarias para que otros líderes vayan asumiendo sus funciones. El copo de nieve en Comunidades se organizó con un grupo de cuatro docentes líderes que se responsabilizaban del monitoreo, ejecución en la escuela de una estrategia específica que podría ser Tertulia Literaria, Grupo Interactivo u otro. Dicho docente hacía un seguimiento mensual a los profesores a su cargo y entregaba los instrumentos de monitoreo al representante del proyecto dos veces al año (junio y noviembre). El acompañante o coordinador tenía una comunicación continua con dicho equipo escolar. Uno de los resultados fue el empoderamiento de los mismos, a tal punto que el equipo Copo de Nieve de la escuela asumía el proceso de capacitación al inicio del año a todos sus compañeros docentes; además, garantizaba una mayor eficacia en recojo de información. Al año siguiente, ellos mismos ya insertaban la estrategia y fomentaban la institucionalización.

Otro ejemplo del alto nivel de compromiso y empoderamiento de estos equipos es que ofrecieron su tiempo y apoyo gratuito fuera de su horario de trabajo, en diversos talleres de capacitación dirigidos a los 115 colegios de Jornada Escolar Completa de Lima Metropolitana para que implementen la Tertulia Dialógica Literaria. Fueron más de 35 docentes de los equipos de copo de nieve que participaron en estos espacios.

\section{El acompañamiento}

Se realizaron algunos cambios en el proceso de adhesión de las escuelas al proyecto para lograr un mayor empoderamiento y compromiso. El proyecto propone que la comunidad docente luego de recibir la capacitación (sensibilización), tenga un momento para tomar la decisión de ser parte 
del mismo con un $90 \%$ de apoyo mayoritario (Elboj, Puigdellivol, Soler y Valls, 2002). Hasta ese momento, el proceso del voto se realizaba de manera pública mediante el conteo de manos levantadas en el taller. Recogiendo los aportes de Robert Slavin, creador del modelo de escuelas charter Success for All (Slavin et al. 1996), recomienda que el proceso de aceptación de un proyecto nuevo debe de realizarse mediante voto secreto; puesto que, "en primer lugar, asegura que el personal de la escuela esté a bordo, dispuesto a dar al programa su mejor esfuerzo. En segundo lugar, silencia efectivamente a la pequeña minoría en cada escuela que se opone a todo. Después del primer año, las escuelas adicionales que no seleccionaron el programa en la primera ronda deberían tener otra oportunidad, pero para entonces ya habrán visto qué tan bien funciona el programa en las escuelas vecinas" (Slavin, 2017). Es por ello que, el año 2017, se introdujo la estrategia de voto secreto. Consistía en que la totalidad de la comunidad docente escribía un "sí" o un "no" en una hoja de papel $y$, en ese momento, se realizaba el conteo de los votos. Con esta estrategia de cada diez colegios, siete u ocho aceptaban implementar la propuesta en toda la escuela. El aspecto positivo es que garantizaba un mayor compromiso de parte de los docentes en la implementación.

Respecto al respeto de la libertad docente para implementar cambios, Fullan (2018), dice que el cambio debe de ser "voluntario, pero inevitable" ( $p$. 24). De esta manera se garantiza una apropiación progresiva para llegar poco a poco a más escuelas y con alto nivel de calidad en la implementación.

\section{Los clusters}

En la línea del desarrollo y fortalecimiento de liderazgos intermedios (Fullan y Boyle, 2014), la estrategia de implementación incluyó un mayor acercamiento con los organismos de gobierno educativo en educación, que en el Perú se llaman Unidades de Gestión Educativa Local (UGEL). Se desarrolló una estrategia de formación y asistencia técnica para la implementación del proyecto a los especialistas o funcionarios de dichas entidades. Se trabajó con las UGEL que mostraron una mayor aceptación a la propuesta. Se denominó a esta estrategia trabajo por clusters. Se entiende por cluster un espacio geográfico donde se aspira tener una expansión a la totalidad 
de escuelas. Las UGEL adheridas fueron las siguientes: La UGEL Sullana en Piura, UGEL 03 en Lima y la UGEL Concepción en Junín. El año 2019 se adhirió la UGEL 08 de la región de Lima Provincias.

Uno de los frutos del trabajo de asistencia técnica a la UGEL fue que favoreció el intercambio de buenas prácticas en las escuelas de la red de su jurisdicción. En el Perú, existe un control sobre el uso del tiempo en los directores, mediante un sistema de visitas sorpresa de un equipo de supervisores de una estrategia Ilamada "semáforo escuela", que se inspiró en el trabajo realizado por Michael Barber en Pakistán (2013). E trabajo cercano con las UGEL permitió que los directivos puedan participar en los encuentros y pasantías inter-escuelas. Se buscaba que dichos encuentros se realizaran en las mismas escuelas para que conozcan la realidad de las mismas de la red (Fullan, 2009; A. Hargreaves y Fink, 2008).

\section{Mirando al futuro:}

\section{Teoría de la acción 2019}

Se descubrió que las escuelas y UGEL padecían de un alto nivel de sobrecarga y fragmentación por la cantidad de iniciativas a las que son sometidos. Es por ello que, para un mejor empoderamiento y difusión del proyecto, se procede a ofrecer asistencia a un proceso de ordenamiento interno para que puedan agrandar adecuadamente los principios y estrategias del proyecto, de manera que sume para el desarrollo de la escuela $y$ no se convierte en un distractor más para la misma. A este proceso de ordenamiento y generación de una comprensión compartida profunda entre los equipos de escuelas y UGEL, de lo que se quiere hacer y cómo se quiere hacer, se denomina coherencia (Fullan y Quinn, 2016). Así mismo, para centrarse en el escalonamiento, se trabajará en dos frentes: profundizar en la transformación de un grupo de escuelas referentes y una mayor incidencia en políticas públicas, por medio de la asistencia técnica permanente a las Unidades de Gestión Educativa Local. Este enfoque ayuda a que el proyecto se inserte de manera integrada en las actividades de la vida de la escuela o de la Unidad de Gestión Educativa Local. La experiencia de estos años muestra que, para una mejor inserción de las estrategias en las escuelas y órganos de gobierno intermedios, es necesario que estos pasen primero por un proceso de ordenamiento y generación de coherencia interna. 
- Se realizará un curso de 50 horas de formación teórico-práctico en el primer semestre.

la que cada hipótesis está conectada a un indicador accionable que permite la validación o no de dicha asunción de acto de fe (Ries 2011). Los indicadores accionables "permiten tomar decisiones de negocio con criterio y establecer las acciones que sean pertinentes. Por el contrario, los indicadores "vanidosos" ofrecen mediciones sesgadas, mostrando el mundo "de color de rosa; pero, no reflejan de forma adecuada los auténticos motores de crecimiento de una empresa" (Ries 2011, p. 117).

Por ello, la teoría de la acción reformulada es la siguiente:

Si las escuelas de referencia, siguiendo el marco de coherencia, aplican con amplitud e intensidad las Actuaciones Educativas de Éxito, se acelerará la mejora de aprendizajes y resultados de convivencia.

- Conformaremos la Comisión Mixta de Aprendizaje de la escuela que implementará el Marco de Coherencia:

- Realizaremos la formación de las Comisiones en el Marco de Coherencia.
Acompañaremos al COMIX en el diseño e implementación del Plan a 100 días de la IE.

- Se hará seguimiento y asesoría al plan a 100 días.

- Se fortalecerán redes entre escuelas mediante encuentros inter-escuelas, Foros regionales, foro nacional, encuentro de red por UGEL (sábados y 4 de mayo), y se realizó feedback de sus planes a 100 días.

- Garantizaremos sostenibilidad con el enfoque de movimiento social y de movilización entre pares.

- Realizaremos las formaciones y capacitaciones lideradas por pares docentes y directivos.

Si formamos y ayudamos a que las UGEL $y$ oficinas del gobierno nacional se organicen en torno al marco de coherencia e inserten orgánicamente las $\mathrm{AEE}$, se garantizará una expansión, sostenibilidad del proyecto $y$, al mismo tiempo, el desarrollo de entidades referentes para el país.

- Si formamos y ayudamos a que las UGEL y oficinas del gobierno 
nacional interesadas apliquen comentarla, discutirla y cuestionarla coherencia en sus organizaciones con datos empíricos (City, et al., 2009) e inserten orgánicamente las AEE, o indicadores accionables (Ries, 2011). garantizaremos una adhesión Los pasos siguientes son empoderar de funcionarios al proyecto y a los grupos de participación escolar, una expansión, sostenibilidad y llamados Comisiones Mixtas, en el entidades referente (modelo) para el país. proyecto. Es por elloque se haconstituido a nivel del equipo líder, los COA,

- Para ello, acompañaremos y pequeñas Comunidades de aprendices haremos de facilitadores en las ugeles respectivas, una vez por semana o más, ofreciendo todas las herramientas y materiales que necesiten (Sullana, 08, 03, Concepción, Paruro, DISER?). conformados por cinco personas máximo, con un líder responsable. Estos buscan ser un espacio para generar alta fluidez de la información para definir qué y cuáles estrategias están funcionando o no en el aula, así mismo, se constituyen

- Crearemos y fortaleceremos una red de organismos intermedios para que se enriquezcan mutuamente mediante encuentros presenciales, pasantías y comunicación virtual continua.

- Tendremos ugeles y entidades que no se sobrecarguen, fragmenten ni generen confusión y parálisis en los directores y docentes.

- Fomentaremos que los funcionarios de órganos intermedios visiten a las escuelas referencias de manera periódica.

Como afirma Elmore, la clave es hacer explícita la teoría de la acción, en espacios de reflexión sobre la acción. En estos espacios se busca generar "aprendizajes validados"(Ries 2011), para ir afinando la mejor implementación del proyecto. Aprendizaje validado se define como aquel aprendizaje empíricamente probado para saber qué quieren o qué necesitan realmente los usuarios o consumidores (Ries, 2011). 


\section{Conclusiones}

La implementación del proyecto Comunidades de Aprendizaje es un proceso dinámico que tiene mayores posibilidades de expandirse con calidad, entendida esta como profundidad, difusión, empoderamiento de la propuesta, en la medida que se tenga teorías de la acción explícitas y abiertas a ser empíricamente refutables.

\section{Sobre el desarrollo de capacidades en los líderes escolares}

Los líderes escolares desarrollan capacidades de trabajo participativo y democrático. Dado que las instituciones educativas públicas del Perú se rigen por normativas, los directivos desarrollan en muchos casos relaciones basadas en las normas, lo que empobrece el acento en las relaciones y vínculos de persona a persona. En ese contexto, el proyecto ayuda a los líderes a generar otro tipo de relación con maestros, estudiantes y padres de familia.

Se desarrolla una cultura de escucha de los estudiantes. Si bien en los documentos el sistema está centrado en el estudiante, en la práctica los líderes escolares tienen pocos espacios para que sus

condiscípulos, desde los más pequeños, puedan expresar sus inquietudes $y$ opiniones. Los líderes escolares aprenden que existen estrategias, como son las Comisiones Mixtas, que les permiten escuchar de primera mano la voz de los estudiantes.

El líder escolar aprende a involucrar a toda la comunidad educativa. Con la aplicación del proyecto el equipo administrativo se involucra mucho más en la labor pedagógica. Estos encuentran sentido a su labor y su relación con el fin pedagógico de la misma. De esa manera, se toma conciencia de que su labor es condición para un aprendizaje de calidad. Esto ocurre mediante la participación de los mismos en las Comisiones Mixtas y en los Grupos Interactivos en los que estos participan como voluntarios. De esta manera, los líderes escolares logran una mayor cohesión de toda la comunidad educativa en función de los aprendizajes.

\section{Sobre el cambio educativo}

El "cambio educativo es un proceso" (Fullan, 2018, p.24).En muchosmomentos se avanza tres pasos y se retrocede dos. Es un proceso y un aprendizaje continuo de parte de los implementadores y los actores involucrados. A continuación, 
se realiza un análisis siguiendo las categorías de Coburn (2003):

Profundidad: Sigue siendo un desafío el cambio de creencias en los docentes. Lo queocurreenalgunoscasosesqueaplican las estrategias de trabajo igualitario en el aula, pero una vez finalizada la estrategia regresan a interacciones que responden a pedagogías no igualitarias. En otros casos, se tiene docentes que han repensado sus prácticas en la medida que han ido aplicando las estrategias del proyecto.

Sostenibilidad: Se han dado grandes avances en el proceso de lograr la sostenibilidad mediante la formación y empoderamiento de los órganos intermedios $y$ algunas oficinas del gobierno nacional. Otro elemento que está favoreciendo que las escuelas mantengan el cambio es el fortalecimiento de las relaciones entre las escuelas de la red de Comunidades de Aprendizaje, mediante encuentros formales e informales. El aprendizaje entre pares ha demostrado su efectividad.

Difusión: Ha sido de gran ayuda el trabajo con los órganos de gobierno intermedio, así como la inserción en la normativa nacional, para proteger las prácticas que se quieren insertar. Así mismo está siendo de gran ayuda, si bien todavía es prometedor, el trabajar con escuelas de referencias en las regiones que se han elegido y que hayan aceptado ser cluster de crecimiento del proyecto. Las escuelas demostrativas reciben visitas y son modelo para otras de convivencia y mejoras de aprendizajes.

Apropiación: La estrategia de copo de nieve ha contribuido a la generación de una mayor apropiación del proyecto en las escuelas. Además, el hacer que las escuelas y equipos docentes sean los responsables de la capacitación a otros colegios, genera un alto sentido de pertenencia y apropiación de la propuesta. El sistema de pasantías favorece el empoderamiento en la misma. Fortalecer y crear redes educativas basadas en el intercambio de actividades pedagógicas facilita el desarrollo de la identidad y sentimiento de pertenencia. El enfoque de cambio educativo como movimiento social ha demostrado tener un potencial para generar identidad y pertenencia (Malone, Rincón-Gallardo y Kew, 2017). 


\section{Referencias}

Alonso, J. G., y Saso, C. E. (2011). El giro dialógico de las ciencias sociales: hacia la comprensión de una metodología dialógica, (12), 77-94.

Aubert, A., Flecha, A., García, C., Flecha, R., y Racionero, S. (2011). Aprendizaje dialógico en la sociedad de la información. Hipatia.

Aubert, A., García, C., y Racionero, S. (2009). El aprendizaje dialógico. Cultura YEducación, 21(2), 129-139.

Barber, M. (2013). The good news from Pakistan. Reform Research Trust, 33.

Bryk, A. S., Sebring, P. B., Allensworth, E., Luppescu, S., y Easton, J. Q. (2010). Organizing schools for improvement: Lessons from Chicago. University Of Chicago Press.

City, Elizabeth A, Elmore, R. F., Fiarman, S. E., y Teitel, L. (2009). Instructional rounds in education: A network approach to improving teaching and learning. ERIC.

Coburn, C. E. (2003). Rethinking scale: fomentan la inclusión. Conclusiones del Proyecto Includ-ed.

Moving beyond numbers to deep and lasting change. Educational Researcher , 32(6), 3-12.

Colbert, V., y Sarmiento, A. (2017). Social justice, educational change and Escuela Nueva. In H. J. Malone, S. Rincón-Gallardo, y K. Kew (Eds.), Future Directions of Educational Change: Social Justice, Professional and System Change.

\section{Routledge.}

Elboj, C., Puigdellivol, I., Soler, M., y Valls, R. (2002). Comunidades de aprendizaje: Transformar la educación. Peru Graó.

Farrell, J., Manion, C., y Rincón-Gallardo, S. (2017). Reinventar la escuela: alternativas radicales exitosas del sur global. REVISTA LATINOAMERICANA DE ESTUDIOS EDUCATIVOS, XLVII(2).

Flecha, R. (2006). Strategies for inclusion and social cohesion in Europe from education. Integrated Project, 6FP European Commission.

Flecha, R. (2010). Prácticas educativas que Proyecto Included. 
Freire, P. (2005). Pedagogía del oprimido. Siglo XXI.

Fullan, M. (2005). Leadership y sustainability : system thinkers in action. Thousand Oaks, Calif.: Corwin Press.

Fullan, M. (2009). Have theory, will travel: A Theory of action for system change. In A. Hargreaves y M. Fullan (Eds.), Change Wars. Bloominghton: Solution Three.

Fullan, M. (2018). Surreal Change. Hargreaves, A. y Fullan, M. (2014). Capital Surreal Change. https://doi. org/10.4324/9781315682952-1

Fullan, M. y Boyle, A. (2014). Big-City School Reforms: Lessons from New York, Toronto, and London. Teachers College Press.

Fullan, M., y Hargreaves, A. (2009). Change Wars. Solution Tree Press.

Fullan y Quinn. (2016). Coherence: The right drivers in action for school, districts and systems. Corwin Press.

Ganz, M. (2009). Why David sometimes wins: Leadership, organization, and strategy in the California farm worker movement. Oxford University Press.
Ganz, M. (2015). Liderazgo, organización, podery acción.

Habermas, J. (1987). Teoría de la acción comunicativa (Vol. 1). Madrid: Taurus.

Hargreaves, A. (2011, May 22). School colaboration.

Hargreaves, A. y Fink, D. (2008). El liderazgo sostenible. Madrid: Morata.

profesional: Transformando la enseñanza en cada escuela. Madrid: Morata.

Malone, H. J., Rincón-Gallardo, S. y Kew, K. (2017). Future Directions of Educational Change: Social Justice, Professional and System Change. Routledge.

Ries, E. (2011). El método Lean Startup: Las claves para aprender emprendiendo. Crown Business Publishing.

Rincón-Gallardo, S. y Elmore, R. (2012). Transforming Teaching and Learning Through Social Movement in Mexican Public Middle Schools. Harvard Educational Review, 82(4), 471-490.

Slavin, R. (2017, February 23). Implementing Proven Programs. Retrieved March 19, 2019, from 
https://robertslavinsblog.wordpress. com/2017/02/23/implementingproven-programs/

Stringfield, S. y Datnow, A. (1998). Introduction: Scaling up school restructuring designs in urban schools. Sage Publications Sage CA: Thousand Oaks, CA.

Valls, R. (2000). Comunidades de aprendizaje: una práctica educativa de aprendizaje dialógico para la sociedad de la información. Universitat de Barcelona.

\section{Para citar este artículo:}

Barrientos, R. (2019)

Interacciones que transforman:

Implementación de Comunidades de Aprendizaje en el Perú durante los años 2014-2018.

Revista Educa UMCH, 13(1). https://doi.org/10.35756/educaumch.v0i13.94 